\title{
Atenção farmacêutica no uso de plantas medicinais com ação anti-hipertensiva em
}

\author{
idosos \\ Pharmaceutical care in the use of medicinal plants with anti-hypertensive action in the elderly \\ Atención farmacéutica en el uso de plantas medicinales con acción antihipertensiva en ancianos
}

Recebido: 01/07/2021 | Revisado: 09/07/2021 | Aceito: 13/07/2021 | Publicado: 23/07/2021

Luiz Otávio Lopes de Carvalho

ORCID: https://orcid.org/0000-0002-0618-3819 Escola Superior da Amazônia, Brasil

E-mail: luiz.lopes1981@hotmail.com

Thiago Saldanha dos Reis

ORCID: https://orcid.org/0000-0003-3631-3220

Escola Superior da Amazônia, Brasil

E-mail: saldanha.thiago@ymail.com

Gleicy Kelly China Quemel

ORCID: https://orcid.org/0000-0003-1280-560X

Escola Superior da Amazônia, Brasil

E-mail: gkcquemel@gmail.com

Daniele de Araújo Moysés

ORCID: https://orcid.org/0000-0001-6956-1381

Núcleo de Pesquisas em Oncologia, Brasil

E-mail: quimica.dani@gmail.com

Natasha Costa da Rocha Galucio

ORCID: https://orcid.org/0000-0003-4923-1478

Núcleo de Pesquisas em Oncologia, Brasil

E-mail: natashagalucio@gmail.com

Regianne Maciel dos Santos Correa

ORCID: https://orcid.org/0000-0002-9837-4304

Universidade Federal do Pará, Brasil

E-mail: regianne@ufpa.br

\begin{abstract}
Resumo
A hipertensão é uma doença cardiovascular de elevada prevalência entre a população de idosos, representando um fator de risco expressivo de morbidade e mortalidade. A partir do conhecimento empírico, grande parte desta população usa as plantas medicinais para tratamento de enfermidades, como a hipertensão. Objetivou-se identificar na literatura, as principais plantas medicinais com potencial anti-hipertensivo utilizadas por idosos no tratamento da hipertensão, destacando a importância da atenção farmacêutica na terapêutica. A pesquisa foi realizada no Scielo, BVS, PubMed e Google Acadêmico no período entre 2011 e 2021, nos idiomas português, inglês e espanhol. Foram selecionados 45 artigos e escolhidas 7 espécies de plantas medicinais com potencial anti-hipertensivo. Allium sativum, Camellia sinensis, Cymbopogon citratus, Ginkgo biloba, Hibiscus sabdariffa, Panax ginseng e Zingiber officinale, apresentaram ações farmacológicas no controle da hipertensão. O uso indiscriminado, destas espécies, pode provocar efeitos adversos como desconfortos gástrico intestinais, hepatotoxicidade e cefaleia, eventos hemorrágicos e cardiovasculares. Além disso, as espécies, Ginkgo biloba, Hibiscus sabdariffa, Panax ginseng e Zingiber officinale apresentaram interações medicamentosas com medicamentos antiplaquetários e/ou anticoagulantes e antiepilépticos. Diante dos riscos apresentados, principalmente em idosos, a atenção farmacêutica representa uma ferramenta em potencial na terapêutica, evidenciados no acompanhamento farmacoterapêutico, orientação na forma do preparo, coleta e armazenamento das plantas medicinais, facilitador de adesão do tratamento, educador e prescritor na prevenção de doenças crônicas como a hipertensão, promovendo o uso racional das plantas medicinais com propriedade anti-hipertensivas.
\end{abstract}

Palavras-chave: Atenção farmacêutica; Hipertensão; Idoso; Plantas medicinais.

\footnotetext{
Abstract

Hypertension is a highly prevalent cardiovascular disease among the elderly population, representing an expressive risk factor for morbidity and mortality. Based on empirical knowledge, a large part of this population uses medicinal plants to treat illnesses such as hypertension. The objective was to identify in the literature the main medicinal plants with anti-hypertensive potential used by the elderly in the treatment of hypertension, highlighting the importance of pharmaceutical care in therapy. The research was carried out in Scielo, BVS, PubMed and Google Academic in the period between 2011 and 2021, in Portuguese, English and Spanish. 45 articles were selected and 7 species of
} 
medicinal plants with antihypertensive potential were chosen. Allium sativum, Camellia sinensis, Cymbopogon citratus, Ginkgo biloba, Hibiscus sabdariffa, Panax ginseng and Zingiber officinale showed pharmacological actions in the control of hypertension. The indiscriminate use of these species can cause adverse effects such as intestinal gastric discomfort, hepatotoxicity and headache, hemorrhagic and cardiovascular events. In addition, the species, Ginkgo biloba, Hibiscus sabdariffa, Panax ginseng and Zingiber officinale showed drug interactions with antiplatelet and/or anticoagulant and antiepileptic drugs. In view of the risks presented, especially in the elderly, pharmaceutical care represents a potential therapeutic tool, evidenced in pharmacotherapeutic monitoring, guidance in the form of preparation, collection and storage of medicinal plants, facilitator of treatment adherence, educator and prescriber in the prevention of chronic diseases such as hypertension, promoting the rational use of medicinal plants with antihypertensive properties.

Keywords: Pharmaceutical care; Hypertension; Elderly; Medicinal plants.

\section{Resumen}

La hipertensión es una enfermedad cardiovascular de alta prevalencia entre la población anciana, que representa un factor de riesgo expresivo de morbilidad y mortalidad. Con base en el conocimiento empírico, una gran parte de esta población utiliza plantas medicinales para tratar enfermedades como la hipertensión. El objetivo fue identificar en la literatura las principales plantas medicinales con potencial antihipertensivo utilizadas por los ancianos en el tratamiento de la hipertensión, destacando la importancia del cuidado farmacéutico en terapia. La investigación se realizó en Scielo, BVS, PubMed y Google Academic en el período comprendido entre 2011 y 2021, en portugués, inglés y español. Se seleccionaron 45 artículos y se eligieron 7 especies de plantas medicinales con potencial antihipertensivo. Allium sativum, Camellia sinensis, Cymbopogon citratus, Ginkgo biloba, Hibiscus sabdariffa, Panax ginseng y Zingiber officinale mostraron acciones farmacológicas en el control de la hipertensión. El uso indiscriminado de estas especies puede ocasionar efectos adversos como malestar gástrico intestinal, hepatotoxicidad y dolor de cabeza, eventos hemorrágicos y cardiovasculares. Además, las especies Ginkgo biloba, Hibiscus sabdariffa, Panax ginseng y Zingiber officinale mostraron interacciones farmacológicas con fármacos antiplaquetarios y/o anticoagulantes y antiepilépticos. Ante los riesgos que presenta, especialmente en los ancianos, la atención farmacéutica representa una potencial herramienta terapéutica, evidenciada en el seguimiento farmacoterapéutico, orientación en forma de preparación, recolección y almacenamiento de plantas medicinales, facilitador de la adherencia al tratamiento, educador y prescriptor en la prevención. de enfermedades crónicas como la hipertensión, promoviendo el uso racional de plantas medicinales con propiedades antihipertensivas.

Palabras clave: Atención farmacéutica; Hipertensión; Anciano; Plantas medicinales.

\section{Introdução}

Desde os primórdios da humanidade, o homem buscava a cura utilizando as plantas medicinais. A conexão entre o homem e sua busca por drogas na natureza data de um passado remoto, do qual há ampla evidência em várias fontes como documentos escritos e em monumentos preservados. A consciência do uso de plantas medicinais é resultado de muitos anos de lutas contra as doenças, devido às quais o homem aprendeu a buscar drogas em cascas, sementes, corpos frutíferos e outras partes das plantas. A ciência contemporânea reconheceu sua ação ativa e incluiu na farmacoterapia moderna uma série de medicamentos de origem vegetal, conhecidos por antigas civilizações e utilizados ao longo de milênios (Locatelli et al., 2017).

$\mathrm{O}$ uso das plantas medicinais no tratamento da hipertensão, faz parte da cultura popular, principalmente entre os idosos, e nas últimas décadas, o interesse pela fitoterapia teve um aumento considerável entre os usuários, pesquisadores e serviços de saúde. Esse crescimento, veio acompanhado do aumento do seu uso abusivo, em que as pessoas de forma aleatória a utilizam para tratamento da hipertensão, como as plantas medicinais como o Panax ginseng, Ginkgo biloba, entre outras. Por serem naturais, acreditam que o seu uso é seguro e sem efeitos colaterais (Shaito et al., 2020).

Esse uso indiscriminado das plantas medicinais, no tratamento da hipertensão, representa um grande perigo, principalmente entre os idoso. Isso porque os riscos são mais expressivos, devido às modificações na fisiologia do corpo idoso. Neste grupo, há uma redução do fluxo sanguíneo e das atividades enzimáticas hepáticas, redução na produção de suco gástrico e na velocidade do esvaziamento gástrico, aumento do teor de tecido adiposo total, perda do teor de água total e da quantidade de proteínas plasmáticas, além da contenção da irrigação renal. Adicionalmente, idosos estão mais expostos a polifarmacoterapia, o que torna mais elevado o risco de interação medicamentosa (Tavares et al., 2018).

Nesse contexto, a atenção farmacêutica representa uma ferramenta em potencial no cuidado com o idoso, que utiliza 
as plantas medicinais e seus derivados para tratar a hipertensão. Atenção farmacêutica é o conjunto de ações centrada no paciente, para promoção, proteção e recuperação da saúde e prevenção de doenças relacionadas ao uso de medicamentos. No Brasil, o conceito de atenção farmacêutica é utilizado para definir um amplo conjunto de ações clínicas promovidas pelos farmacêuticos, havendo duas formas de tradução do termo: "atenção farmacêutica" ou "cuidado farmacêutico". Ambos os termos estão diretamente relacionados ao papel clínico do farmacêutico na melhoria da qualidade de vida do paciente (Pelentir, Deuschle \& Deuschile, 2015).

Portanto, o presente trabalho tem como objetivo identificar, através de revisões bibliográficas, as principais plantas medicinais com potencial anti-hipertensivo utilizadas por idosos no tratamento da hipertensão, destacando a importância da atenção farmacêutica na terapêutica.

\section{Metodologia}

O estudo foi desenvolvido através de um levantamento bibliográfico de caráter exploratório e método qualitativo (Pereira et al., 2018), acerca da atenção farmacêutica no uso das plantas medicinais com ação anti-hipertensiva, direcionado ao idosos. As pesquisas foram realizadas através de artigos científicos publicados no Scientific Electronic Library Online (Scielo), Biblioteca Virtual de Saúde (BVS), US National Library of Medicine/NationalInstitutes Of Health (PubMed) e plataforma Google Acadêmico e documentos oficiais. Como estratégia de busca de artigos, foram utilizados os seguintes descritores em cruzamento (quadro 1): phytotherapy (fitoterapia), herbal (ervas), plants (plantas), "medicinal plants" (plantas medicinais), "antihypertensive" (anti-hipertensivo), "hypertensive" (hipertensivo), "blood pressure" (pressão arterial), "hypertension" (hipertensão), "active principle" (princípio ativo), "elderly" (idoso), "drug interactions" (interações medicamentosas), "bioactive phytochemical" (fitoquímico bioativos), "side effects" (efeito colaterais) e "mechanism of action" (mecanismo de ação). Esses descritores foram aplicados na busca de acordo com as particularidades de cada base de dados.

Quadro 1 - Descritores e operadores booleanos utilizados na busca.

\begin{tabular}{|c|}
\hline Origem de dados - Descritores \\
\hline 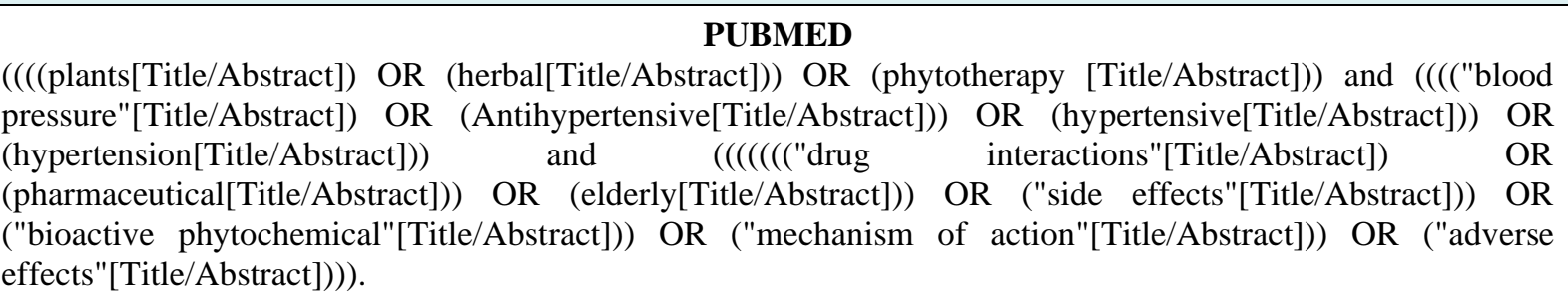 \\
\hline $\begin{array}{c}\text { SCIELO } \\
\text { (ti:("plantas medicinais")) OR (ti:("atenção farmacêutica")) }\end{array}$ \\
\hline $\begin{array}{c}\text { BVS } \\
\text { ti:(("plantas medicinais") OR ("atenção farmacêutica")) }\end{array}$ \\
\hline $\begin{array}{c}\text { GOOGLE ACADÊMICO } \\
\text { "atenção farmacêutica" + "plantas medicinais" }\end{array}$ \\
\hline
\end{tabular}

Fonte: Autores.

No refinamento 01 da pesquisa (Figura 1), foram selecionados os artigos pelo título/resumo, artigos completos gratuitos, entre 2011 a 2021, nos idiomas português, inglês e espanhol. No refinamento 02 foram selecionados os artigos que tratassem especificamente do tema da pesquisa e foram utilizados aqueles que continham informações relevantes da resposta do problema e desenvolvimento do trabalho, porém o embasamento não se restringiu a busca sistemática de artigos, sendo 
incluídas publicações oficiais com conteúdo relevante para complementar a pesquisa e para o enriquecimento da discussão sobre o tema.

Figura 1 - Fluxograma da Metodologia de Busca de Artigos.

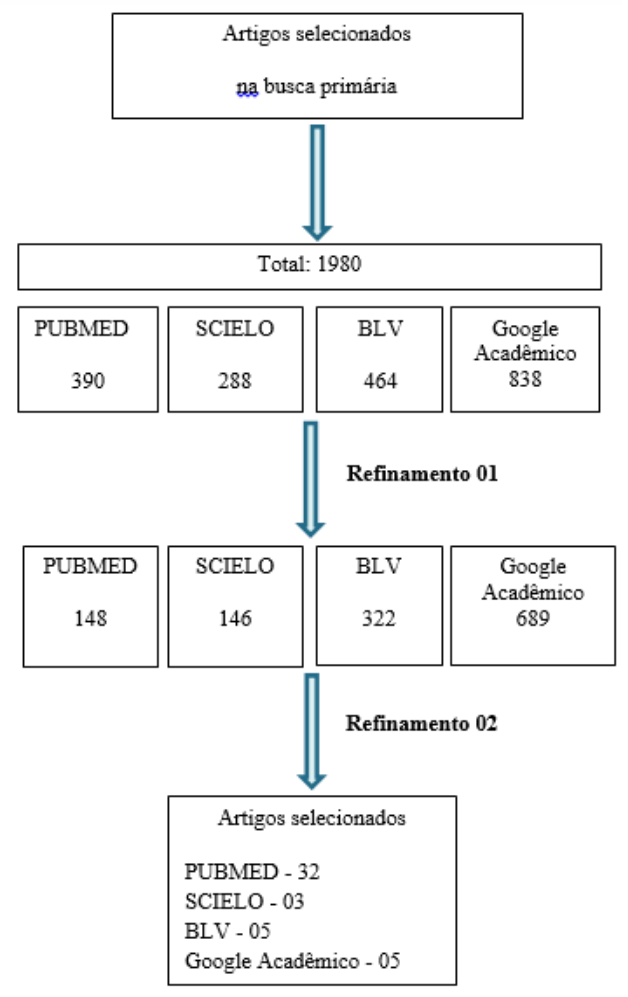

Fonte: Autores.

\section{Resultados e Discussão}

A partir dos artigos selecionados, foram escolhidas 7 espécies de plantas medicinais com potencial anti-hipertensivo, seguindo como critério, aquelas espécies mais citadas em artigos que apresentavam estudos científicos consistentes, comprovando suas propriedades hipotensivas apresentadas no quadro 2 . 
Quadro 2 - Plantas Medicinais com Potencial anti-hipertensivo.

\begin{tabular}{|c|c|c|c|}
\hline Plantas medicinais & Principais Princípios Ativos & Ação farmacológica & Referências \\
\hline $\begin{array}{l}\text { Allium sativum } \\
\text { (Alho) }\end{array}$ & $\begin{array}{c}\text { Alicina } \\
\gamma \text {-glutamil- } S \text { - alilcisteína }\end{array}$ & $\begin{array}{l}\text { Inibidor do ECA } \\
\text { Vasodilatadores }\end{array}$ & $\begin{array}{c}\text { Nakasone et al. (2013); } \\
\text { Ried (2016) }\end{array}$ \\
\hline $\begin{array}{c}\text { Camellia sinensis } \\
\text { (Chá verde, chá preto ou } \\
\text { chá oolong) }\end{array}$ & Catequinas & Vasodilatadores & Peng et al. (2014) \\
\hline $\begin{array}{l}\text { Cymbopogon citratus } \\
\text { (Capim limão) }\end{array}$ & Citral & $\begin{array}{c}\text { Vasodilatadores } \\
\text { Bloqueador de cálcio }\end{array}$ & Devi et al. (2012) \\
\hline $\begin{array}{c}\text { Ginkgo biloba } \\
\text { (Árvore-avenca) }\end{array}$ & Flavonoides e terpenóides & $\begin{array}{l}\text { Vasodilatadores } \\
\text { Inibidor do ECA }\end{array}$ & Mansour (2011) \\
\hline $\begin{array}{l}\text { Hibiscus Sabdariffa } \\
\text { (Hibiscus) }\end{array}$ & Antocianinas & $\begin{array}{l}\text { Vasodilatadores } \\
\text { Bloqueador de cálcio } \\
\text { Inibidor do ECA }\end{array}$ & Hopkins et al. (2013) \\
\hline $\begin{array}{c}\text { Panax ginseng } \\
\text { (Ginseng vermelho) }\end{array}$ & Ginsenosídeos & Vasodilatadores & $\begin{array}{c}\text { Jovanovski et al. (2014); } \\
\text { Kim (2012) }\end{array}$ \\
\hline $\begin{array}{l}\text { Zingiber officinale } \\
\text { (Gengibre) }\end{array}$ & $\begin{array}{l}\text { Compostos fenólicos e } \\
\text { terpênicos }\end{array}$ & $\begin{array}{c}\text { Inibidor do ECA } \\
\text { Vasodilatadores }\end{array}$ & Akinyemi et al. (2015) \\
\hline
\end{tabular}

Legenda: ECA: Enzima Conversora de Angiotensina. Fonte: Autores.

\subsection{Plantas Medicinais com Potencial anti-hipertensivo}

\subsubsection{Allium sativum (Alho)}

Ao longo da história antiga o alho é conhecido por suas propriedades medicinais. Estudos mais recentes indicam que o Allium sativum tem potencial para reduzir a pressão arterial em indivíduos hipertensos. Além disso, colaboram na redução da concentração de colesterol quando está elevado e atuam no fortalecimento do sistema imunológico (Ried, 2016). O alho é muito utilizado nos alimentos, na forma em pó, in natura, óleo e em extrato, seus constituintes principais são organossulfurados como a alicina (principal constituinte ativo), ajoene, S-alil- 1- cisteína, dissulfetos de dialila (DADS), tiossulfonato de metila e trissulfetos de dialila, que são responsáveis pelo efeito seu farmacológico (Al Disi, 2016). Segundo Nakasone et al. (2013), alicina e $\gamma$-glutamil- S- alilcisteína (GSAC) são os responsáveis por esse efeito hipotensor, estes atuam inibindo a ECA (enzima conversora de angiotensina) e induzindo relaxamento dependente e independente do endotélio, provocando uma vasodilatação. Outros estudos identificam vasorelaxamento em artérias pulmonares de ratos quando utilizaram extrato de alho, efeito explicado pela biodisponibilidade de NO (óxido nítrico) (Ku, 2002). Em estudos clínicos com suplementação a base de alho na dieta, por 12 semanas, reduziu de forma considerável a PAS (Pressão Arterial Sistólica) e a PAD (Pressão Arterial Diastólica) nos pacientes com hipertensão. Após o término desse período de suplementação, nas 4 semanas seguintes, ainda há efeitos hipotensivos substanciais (Nakasone et al., 2013). O alho é considerado seguro, apresentando leves desconfortos gástrico intestinais geralmente ocasionado por intolerância. Geralmente, é ocasionado pelo alho cru, que pode ser substituído pelo alho envelhecimento que atenua os efeitos (Varshney \& Budoff, 2016). Segundo Gurley et al. (2012), a maioria dos suplementos de alho apresentam risco limitado de produzir interação medicamentosa, com importância clínica.

\subsubsection{Camellia sinensis (Chá verde, oonlong, preto)}

Desde o século X a.c havia relatos do valor medicinal das folhas Camellia sinensis. Estas são processadas para obter o chá verde não fermentado, chá oolong parcialmente fermentado e o chá preto fermentado. O principal constituinte do chá são as catequinas, formadas por epicatequina, epigalocatequina, galato de epicatequina e galato de epigalocatequina. Estudos sugerem que essas substâncias apresentem ação farmacológica benéficas em doenças cardiovasculares como a hipertensão 
(Deka, 2011). Apesar do chá apresentar cafeína que possui características excitantes, estudos de meta-análise envolvendo 1.367 indivíduos, indicaram o chá verde como potencial hipotensor, reduzindo a PAS em -1,98 mmHg e PAD em -1,92 mmHg (Peng et al., 2014). Estudo epidemiológico transversal, associa o chá verde e o chá preto ao menor risco de hipertensão (Li et al., 2016). Na China, estudos de coorte indica que o chá, em destaque o chá verde, apresenta benefício em paciente idosos nãohipertensos ou naqueles que estão usando medicamentos anti-hipertensivos (Yin et al., 2017). O chá verde pode reduzir a pressão arterial por diferentes mecanismos, como manter o equilíbrio entre os fatores vasoconstritores, vasodilatadores e hiperpolarizantes. Tem um efeito crescente na produção de NO para aumentar a função ventricular e controlar a produção de ROS (Espécie Reativas de Oxigênio), produzindo enzimas antioxidantes e reduzindo as enzimas pró-oxidantes (Peng, 2014). Pesquisas revelam que o chá verde é considerado seguro em altas doses e preparações. Porém elevadas doses podem gerar efeito crítico de hepatotoxicidade, ocasionado pelas catequinas, como a epigallocatechin gallate. Uma dose de até $704 \mathrm{mg} / \mathrm{dia}$ de galato de epigalocatequina na preparação de chá em bebidas é considera segura (Hu et al., 2018).

\subsubsection{Cymbopogon citratus (Capim limão)}

O extrato do $C$. citratus é utilizado mundialmente na alimentação, cosméticas e em tratamento de diversas doenças. Estudos tem identificado que esta planta possui propriedades cardiovasculares, antimicrobianas, antifúngicas, antiinflamatórias, antineoplásicas, antidiabéticas, antioxidantes, cardiovasculares, inseticidas e pesticidas (Kouame, 2016). Tradicionalmente o C. citratus são usados como chás ou decocção seus compostos bioativos também são aplicados como repelente de insetos. O seu cheiro de limão característicos é devido a presença do citral que é o seu principal constituinte. Além deste, possui flavonóides, óleos essenciais, compostos fenólicos, que juntos são responsáveis pelas propriedades farmacológicas (Tajidin, 2012). Segundo Devi (2012), estudos pré-clínicos com ratos, os extratos metanólicos das folhas, caule e raiz de $C$. citratus, apresentaram características de vasodilatação nas contrações estimulada por efedrina. Esse efeito é devido ao seu principal constituinte, citral, que é responsável pela liberação NO, que produz vasorelaxamento inibindo a produção de (S)-2-Amino-5-guanidinopentanoic acid (L-arginina). Além disto, pode atuar como bloqueador em canais de cálcio e afetar a síntese de prostaglandina, desencadeando um efeito vasodilatador. O C. citratus apresentam baixa toxicidade apesar de possuir em sua constituição, cumarinas, taninos, antraquinonas e saponina que representam toxicidade a planta. $\mathrm{O}$ que sugere cautela em indivíduos com problemas de saúde, como renal ou hepática, mulheres grávidas, lactantes e menores de 4 anos (Ekpenyong, Akpan \& Nyoh, 2015).

\subsubsection{Ginkgo biloba (Árvore-avenca)}

O G. biloba é uma espécie medicinal de origem milenar que apresenta grande resistência aos fatores de estresse ambiental, por isso é chamado de fóssil vivo. Devido a sua grande quantidade de compostos bioativos, vem despertando interesse em pesquisas. Seus principais constituintes são flavonoides, (glicosídeos ginkgo-flavona), terpenóides (ginkgolídeos e bilobalídeos), biflavonas e ácidos orgânicos. Os flavonoides do G. biloba são formados por glicosídeos como o caempferol, quercetina e isorhamnetina (Isah, 2015). Extrato de G. biloba a apresenta vários fins terapêuticos, como a redução do fluxo sanguíneo cerebral, proteção dos neurônios colabora na cognição, melhorando problemas de memória e atenção (Mashayekh et al., 2011; Mahmoudian- Sani et al., 2017; Tan et al., 2015). Além disso, apresenta ação antioxidante, colaborando em eliminar o excesso e a produção de radicais livres (Shaito et al., 2020). Em relação a hipertensão, também apresentou efeitos benéficos, pois possui propriedade vasodilatadoras, inibidora do ECA, ativação das vias colinérgicas e melhora a saúde do endotelial. Sua ação hipotensiva ocorre através da ativação do M2 receptores muscarínicos via do NO e da sinalização colinérgica durante a hipertrofia cardíaca (Xiong et al., 2014). Em ensaios com ratos, utilizando extrato de G. biloba, foi constatado a restauração da atividade do eNOS (óxido nítrico sintase endotelial), responsável pela maior parte produção de NO, propiciando uma elevação 
de NO, obtendo um efeito vasodilatador (Mansour et al., 2011; Mesquita et al., 2017). Segundo Xiong et al. (2014), através de ensaios clínicos, não há evidência convincentes para apoiar o uso do G. biloba no tratamento da hipertensão, sendo necessários realizar mais estudos clínicos randomizados para apoiar ou refutar o seu uso. Possíveis interações medicamentosas com antiepilépticos, ácido acetilsalicílico, diuréticos, ibuprofeno, risperidona, rofecoxibe, trazodona e varfarina. Além disso, apresenta efeito de redução plasmática com omeprazol, ritonavir e tolbutamida (Izzo \& Ernst, 2009).

\subsubsection{Hibiscus sabdariffa (Hibiscus)}

As partes dessa planta como flores, folhas e cálice, são utilizadas para elaboração do extrato. É conhecido mundialmente pelo seu uso em bebida quentes ou frias, possui aspecto decorativo e de sabor agradável. Além disso, é utilizado para fins terapêuticos e na culinária. Na Nigéria a infusão do cálice é utilizada devido aos seus efeitos anti-hipertensivos. Estudos experimentais demonstram que o H. sabdariffa possui propriedades antimicrobiana, antioxidante, anticolesterol, antihipertensiva (Abubakar, 2015). Os constituintes dessa planta responsáveis pela ação farmacológica, são as antocianinas, predominantemente cianidina-3-sambubiosídeo e delfinidina-3-sambubiosídeo. Estudos experimentais em animais e humanos demonstram seus efeitos anti-hipertensivo. Utilizando extrato metanoico de $H$. sabdariffa na reatividade vascular em ratos hipertensos, demonstram efeito vasodilatador nos anéis aórticos isolados de ratos. Isso acontece pela via óxido nítrico-cGMP (Monofosfato cíclico de guanosina) relaxante derivada do endotélio e pela inibição do cálcio ( $\mathrm{Ca}^{2+}$ ) (Ajay, 2007). Em estudos experimentais foi constatado que o chá provou uma redução na pressão arterial apresentando uma eficácia semelhante ao captopril, mas menos eficaz que o lisopril (Herrera, 2007). As antocianinas presentes no cálice do hibisco, é considerada a fitoquímica responsável pelo efeito anti-hipertensivos e hipocolesterolêmicos, outros como polifenóis e do ácido de hibisco também apresentam efeito hipotensor. $\mathrm{O}$ mecanismo que explica esse efeito está relacionado aos efeitos antioxidantes da inibição das antocianinas da oxidação do LDL-C (colesterol de lipoproteína de baixa densidade). Além disso, em estudos com animais, apresentou efeito redutor no colesterol total e trigilicérideos. Não foram relatados em estudos os efeitos adversos ou efeitos colaterais, sua toxicidade é considerada de grau baixo (Hopkins et al., 2013). Em estudos pré-clínicos em ratos foi constatado que doses acima de $300 \mathrm{mg} / \mathrm{kg}$ por dia de extrato de $H$. sabdariffa durante 3 meses, foi constatado efeito adverso nas enzimas hepáticas, sugerindo que em doses muito altas o extrato poderia ser hepatotóxico (Fakeye, 2009). Ensaios com ratos e coelhos constaram que interações medicamentosas entre o Hibisco, apresentou ação diurética, com hidroclorotiazida, um medicamento diurético, potencializando o seu efeito. Outra interação ocorreu com o paretamol, pelo efeito farmacocinético, aumentando a eliminação do paracetamol no organismo, o que sugere que paracetamol deve ser consumido 3 a 4 horas do consumo do hibisco (Kolawole, 2004).

\subsubsection{Panax ginseng (Ginseng asiático)}

Desde tempos antigos o ginseng é utilizado tradicionalmente para fins terapêuticos contra diversas doenças. Mais de 12 espécies foi identifica embora para fins terapêuticos as mais utilizadas são $P$. ginseng e o P. quinquefolius (ginseng americano). Esta planta é utilizada para melhorar a inteligência, impotência e hemorragias, para fins de relaxamento e retarda o envelhecimento (Liu, 2020). Além disso, segundo Won et al. (2019), através de ensaios clínicos, demonstrou apresentar propriedades afrodisíacas, anti-inflamatórias, anticâncer (pulmão, fígado, intestino e estômago), antidiabético, cardioprotetor, gastroprotetor, anti-amnéstico e antioxidante (coração e rim). Os constituintes de $P$. ginseng é responsável pela ação farmacológicas são ginsenosídeos, polissacarídeos, peptídeos, alcalóides, poliacetileno e compostos fenólicos. Foi constatado que esta planta possui propriedades hipotensivas (Mucalo et al., 2013; Shin et al., 2013; Shehabi, 2016), porém segundo Kim (2012), o P. ginseng identificou propriedade hipertensivas. Para Jang et al. (2011), esta planta possui efeito bifásico, que depende da concentração, doses mais baixas aumentam a pressão arterial, enquanto doses altas reprimem a pressão arterial. Os 
efeitos hipotensivos do $P$. ginseng são devidos a produção de NO derivado das células endoteliais vasculares por meio da conversão de L-arginina em L-citrulina pelo eNOS. Alguns ginsenosídeos podem ativar e estimular a produção de NO desencadeando a vasodilatação, reduzindo a pressão arterial (Kim, 2018). Em ensaios clínicos, a combinação do extrato de ginseng asiático com a terapia convencional em pacientes diabéticos com hipertensão concomitante diminuiu a rigidez arterial e atenuou a PAS (Mucalo et al., 2013). Em outro ensaio, constatou que o Ginsenoside rg3 do extrato de ginseng vermelho coreano reduz a pressão arterial central e periférica em adultos saudáveis (Jovanovski et al., 2014). A eficácia do ginseng no controle da hipertensão está bem documentada quando combinado com medicamentos hipotensores convencionais (Shaito, 2020). Sobre os efeitos adversos, estudos in vitro e in vivo, bem como ensaios clínicos em humanos, reconheceram que extratos de ginseng têm efeitos colaterais de pouca importância (Park et al., 2014). Porém há relatos de vários casos que levaram a potenciais efeitos colaterais relacionados a eventos cardiovasculares, como aumento da pressão arterial quando utilizado de forma prolongada e indiscriminada (Coon \& Ernst, 2002). Foi relatado que o ginseng interage com várias drogas, mas sua interação com a varfarina, que é um poderoso anticoagulante (medicamento para afinar o sangue) é a mais documentada (Chua et al., 2015). Estudos identificaram interação medicamentosa com midazolam e fexofenadina reduzindo o seu efeito (Malati et al., 2012).

\subsubsection{Zingiber officinale (Gengibre)}

É uma planta perene nativa das ilhas do pacífico, sua raiz além de ser utilizado na culinária como tempero e conhecida pelas suas propriedades terapêuticas. Estudos farmacológicos reconhecem as propriedades medicinais da raiz desta planta, como melhora na digestão, circulação sanguínea, redução lipídica do sangue, atua positivamente da redução da glicose, aliviar a estimulação vestibular e fornecer efeitos anti-inflamatórios, antitumorais, antimicrobianos e antioxidantes (Wang, 2017). Seus constituintes ativos são compostos fenólicos como o gingerol, gingerdiol shogoal, paradóis e os terpênicos ( $\beta$-bisaboleno, curcumin, zingibereno, $\alpha$-farneseno e $\beta$-sesquifelandreno) (Stoner, 2013; Yeh, 2014). O extrato aquoso de gengibre pode reduzir a enzima conversora da angiotensina-1 (ACE) e a peroxidação lipídica, um efeito benéfico no combate a hipertensão (Akinyemi, 2015). Segundo Ojulari et al. (2014) o uso de Zingiber officinale pode reduzir a PA. O gengibre foi citado em muitos artigos, reconhecendo que pode ser usado com medicamentos anti-hipertensivos para o tratamento da hipertensão (Shaban, 2017). O gengibre apresentou atividade inibidora da (ACE) e de arginase que aumenta o nível de NO, provocando a vasodilatação. Em estudos pré-clínicos em ratos utilizando o extrato de gengibre, foi constatado uma redução na pressão arterial (Akinyemi, 2015). Um estudo transversal relacionou a hipertensão e doenças coronárias com a ingestão diária de gengibre, foi evidenciado que os indivíduos que faziam uso do gengibre diariamente tinham menor probabilidade de adquirir essas doenças (Wang et al., 2017). A raiz de gengibre quando consumidas em quantidades acimas de $6 \mathrm{~g}$ pode exacerbar distúrbios gastrointestinais, como refluxo gastrointestinal, azia e diarreia. Pode potencializar os efeitos anticoagulantes e causar sangramento e arritmia em um pequeno número de casos. Além disto, aumenta a secreção de ácido biliar, podendo agravar a formação de cálculos biliares (Ryan, 2014). Este trabalho foi possível constatar a escassez de artigos com ensaios consistentes em português e espanhol, originados da américa latina, que abordem as plantas medicinais com potencial hipotensivo. Os artigos em inglês, oferecido pela Biblioteca Nacional de Medicina dos Estados Unidos através da Pubmed, foram os que apresentaram pesquisas mais promissoras e consistentes na área, embora em grande parte, limitavam-se a estudos pré-clínicos. Isso sugere a necessidade de mais investimentos em pesquisa nessa área, para que avancem em sua maioria para estudos clínicos, o que exige critérios mais rígidos de segurança e eficácia.

\subsubsection{Ação Farmacológica das Plantas Medicinais}


A ação farmacológica das plantas medicinais selecionadas (quadro 3) usadas para o controle da hipertensão, são ocasionadas pela presença de constituintes fitoquímicos bioativos (Verma et al., 2021) que assim como na medicação alopática podem melhorar a saúde e aliviar os sintomas da hipertensão por efeito de adição ou sinergia através de mecanismo de inibidor do ECA, vasodilatador e bloqueador de canais de cálcio (Basati et al., 2021). Em relação as plantas medicinais com ação antihipertensivas em estudo, $100 \%$ apresentaram ação vasodilatadora, ocasionada pela liberação de NO. As espécies responsáveis por inibir a enzima conversora da angiotensina (e a conversão subsequente de angiotensina I em angiotensina II), corresponderam a 57,1\% e apenas 28,5\% apresentaram ação nos bloqueadores de canais de cálcio (Jacobs et al., 2020). Diante dos resultados obtidos é possível constatar que as plantas medicinais selecionadas apresentam evidências científicas de suas propriedades hipotensivas, apresentando a vantagem de ser uma terapêutica de baixo custo e possuir efeitos adversos e interações medicamentosas reduzidas, comparado aos medicamentos alopáticos (Shaito et al., 2020). Apesar das vantagens das plantas medicinais no tratamento da hipertensão, é necessário cautela no seu uso. Isso porque, os seus numerosos metabólicos secundários apresentam complexos mecanismos de ação, com efeitos adversos e interações medicamentosas que ainda não estão completamente elucidados na literatura científica, além disso, fatores como a falta de padronização da preparação da planta (por exemplo, cru, cozido e macerado), afetarão diretamente na dosagem necessária. Outro ponto, é a escolha da parte da planta e sazonalidade em que é colhido, visto que apresentam diferenças fitoquímicas consideráveis (Anwar, 2016).

Quadro 3 - Ação farmacológica das plantas medicinais anti-hipertensivas.

\begin{tabular}{|c|c|c|c|}
\hline $\begin{array}{c}\text { Plantas medicinais com ação } \\
\text { anti-hipertensiva }\end{array}$ & \multicolumn{2}{|c|}{ Ação Farmacológica anti-hipertensiva } \\
\hline & $\begin{array}{c}\text { Inibidor da Enzima } \\
\text { Conversora da } \\
\text { Angiotensina (ECA) }\end{array}$ & Vasodilatador & $\begin{array}{c}\text { Bloqueadores dos canais } \\
\text { de cálcio }\end{array}$ \\
\hline Allium sativum & $\mathrm{x}$ & $\mathrm{x}$ & \\
\hline Camellia sinensis & & $\mathrm{x}$ & $\mathrm{x}$ \\
\hline Cymbopogon citratus & $\mathrm{x}$ & $\mathrm{x}$ & $\mathrm{x}$ \\
\hline Ginkgo biloba & $\mathrm{x}$ & $\mathrm{x}$ & \\
\hline Hibiscus Sabdariffa & $\mathrm{x}$ & $\mathrm{x}$ \\
\hline Panax ginseng & & \\
\hline Zingiber officinale & & \\
\hline
\end{tabular}

Fonte: Autores.

\subsection{Atenção Farmacêutica no Uso das Plantas Medicinais com Ação Anti-hipertensiva Direcionada à Idosos}

Ao realizar pesquisas de artigos, usando o termo "pharmaceutical care" (cuidado farmacêutico) com "medicinal plants" (plantas medicinais) e "Phytotherapy" (fitoterapia) em buscadores de artigos como Pubmed, e BVS, atendendo os critérios de metodologia adotado neste trabalho, apresentaram como resultado apenas um artigo em cada buscador (pelo qual não atendeu ao problema de pesquisa) e no Scielo não apresentou nenhum resultado. Isso demonstra uma grande escassez de literatura científica que contemple as necessidades do farmacêutico no aprimoramento do seu conhecimento na atenção farmacêutica, voltadas para a fitoterapia/plantas medicinais. Diante dessa realidade, foram utilizadas pesquisas no Google Acadêmico adotando outras revistas científicas. Diante das plantas medicinais selecionadas para estudo nesse trabalho, é possível destacar, através de evidências científicas, suas propriedades hipotensivas que podem colaborar na terapêutica, porém semelhante aos medicamentos alopáticos, foram identificados efeitos adversos e interações medicamentosas com menor intensidade. $\mathrm{O}$ uso indiscriminado dessas plantas representa um grande risco a população, principalmente aos idosos, que geralmente fazem uso da polifarmácia e apresentam maior fragilidade em suas funções fisiológica (Tavares et al., 2018). Com preocupação diante do cenário real dos idosos da atualidade, há uma grande necessidade de um plano para uma intervenção 
responsável na estratégia terapêutica deste grupo que busca solução em métodos complementares principalmente em plantas medicinais para resolução dos seus problemas de saúde. Diante disso, cabe ao farmacêutico usar de sua principal função para o desenvolvimento da melhoria na conduta terapêutica com atualizações constantes com o propósito de alcançar junto a equipe de saúde a melhor forma de garantir a qualidade de vida de seus pacientes com a realização da avaliação da farmacoterapia e seus possíveis problemas a fim de promover o melhor proveito desses recursos visto que apesar do conhecimento que os idosos têm a respeito de plantas medicinais a ocorrência de uso com frequentes erros no manuseio, identificação das espécies e posologia ainda é muito recorrente, por isso há uma imensa necessidade da figura farmacêutica (Oliveira et al., 2015). Neste contexto, a atenção farmacêutica representa uma ferramenta em potencial que pode garantir e contribuir com a promoção do uso racional das plantas medicinais no tratamento da hipertensão. Na fitoterapia, exigirá do farmacêutico conhecimento científico, popular e tradicional do uso de plantas medicinais e fitoterápicos, como também das várias formas de uso, de forma a integrar os diferentes saberes, profissionais e usuários, buscando avaliar e garantir a segurança, a eficácia e a efetividade do uso desses recursos terapêuticos (Brasil, 2012). A prática da atenção farmacêutica tem se mostrado de uma expressiva eficácia, tendo impactado na diminuição de agravos de saúde e internações por se tratar de um esquema arquitetado clinicamente de forma excepcionalmente personalizado ao paciente e que busca no profissional atuante o domínio dos dados reais apurados em literaturas para uma expressivo ação direcionada aos grupos mais susceptíveis as problemáticas relacionadas aos agravos e seus principais causadores (Viana, Arantes \& Ribeiro, 2017). Considerando as variáveis que envolvem a hipertensão e o atendimento ao idoso, há a necessidade de vários profissionais atuando em conjunto para tratá-lo, o farmacêutico pode atuar de diversas maneiras para auxiliar o paciente: facilitador da adesão ao tratamento, auxiliar o paciente junto aos fatores de risco (obesidade, tabagismo, ingestão de sal colesterol elevado), auxiliar do prescritor a controlar o tratamento, agente elaborador de campanhas junto à comunidade, auxiliar para amenizar os fatores que dificultam o tratamento, articulação com outros profissionais da saúde e descrição de reações adversas (WHO, 2014; Pelentir, Deuschle \& Deuschle, 2015). Sendo assim, as informações apresentadas nesse trabalho irão colaborar para que o farmacêutico ofereça as corretas orientações ao paciente sobre o uso racional dessas plantas medicinais e seus derivados, sendo necessário associar outras pesquisas e documentos oficiais com informações sobre dose, posologia, coleta, armazenamento e preparo, respeitando as características de cada planta medicinal. Para promover o uso racional de plantas medicinais, são necessários que o farmacêutico realize pesquisas em compêndios oficiais/não oficiais, literaturas científicas consolidadas na área e informações de usos populares/tradicionais com o propósito de validar o uso terapêutico de determinada espécie vegetal e/ ou medicamento fitoterápico. A tradição no uso é legalmente aceita para comprovação de segurança e efetividade de fitoterápicos desde 2004 a partir da RDC No 48 de 2004. A legislação atual para essa finalidade é a RDC no 26 de 2014 da ANVISA (Brasil, 2012; Soares et al., 2021). De posse dessas informações, é necessário colocar em prática a atenção farmacêutica através do método de processo racional de tomada de decisão, para análise da farmacoterapia de pacientes, que fazem uso de plantas medicinais, em que o profissional farmacêutico a partir da abordagem ao paciente, avalia se a planta medicinal em uso pelo paciente é indicado para tratar sua condição de saúde, verificando a efetividade para tal problema/objetivo, se é seguro para o paciente, e por último, se o seu uso está sendo feito da forma mais conveniente para aquele indivíduo (Oliveira et al., 2017). Nessa perspectiva, o paciente é visto de forma holística e integrada, o que se alinha com a perspectiva e filosofia das práticas integrativas e complementares, como a fitoterapia, que busca compreender o indivíduo como um todo e de forma multidisciplinar possibilitando uma abordagem completa e mais efetiva (Telesi, 2016). Na avaliação e acompanhamento de pacientes durante a atenção farmacêutica, praticado através do Gerenciamento da Terapia Medicamentosa (GTM), comumente utilizada nos medicamentos convencionais, geralmente as plantas medicinais são colocadas em um segundo plano, gerando uma lacuna de informações em relação a implementação desse serviço clínico, dados sobre os resultados clínicos, humanísticos, econômicos e sobre a experiência subjetiva dos pacientes em uso da fitoterapia (Ramalho, 2011). Durante a avaliação/consulta o farmacêutico 
precisa estar atento a associação de medicamentos alopáticos com associações com plantas medicinais e fitoterápicos, isso porque geralmente os pacientes não informam que fazem uso da fitoterapia, pois acreditam que por tratarem essas terapêuticas como componentes culturais da sua rotina e comunidade, e por também considerá-los instrumentos terapêuticos que não envolvem riscos à saúde (Marques et al., 2011). Outro componente da prática da atenção da farmacêutica que auxilia o profissional a garantir um manejo da terapia medicamentosa efetivamente centrado no paciente é o reconhecimento da experiência subjetiva com o uso de medicamentos convencionais, fitoterápicos e plantas medicinais. A experiência e os eventos que o paciente vivencia nesse uso faz parte do processo individual e subjetivo do paciente, e que perpassa a sua compreensão em saúde, da sua farmacoterapia, das suas crenças e anseios, que são traduzidos em significados e ações, sejam positivas e/ou negativas (Ramalho, 2011). Essa subjetividade é fundamental para a prevenção, identificação e resolução dos problemas relacionados a medicação (PRM) para construção de um plano de cuidado individualizado, visto que as plantas medicinais e fitoterápicos podem interferir na medicação alopática como no tratamento da hipertensão. Compreender o significado e contexto do uso das plantas medicinais e fitoterápicos é também de suma importância para garantir uma farmacoterapia indicada, efetiva, segura e conveniente para o paciente e tratando da fitoterapia, o seu uso vai além do valor terapêutico. Isso porque está relacionado com componente cultural e tradicional do paciente e da sua comunidade, representando as diversidades e histórias desses. A experiência que o paciente vivencia nesse contexto irá refletir os significados que o mesmo atribui as plantas medicinais para o tratamento e prevenção de seus problemas de saúde. Compreender a experiência subjetiva do paciente que faz uso de plantas medicinais pode guiar o profissional farmacêutico no manejo adequado da terapia medicamentosa de forma centrada no paciente (Figueredo, 2014).

\section{Conclusão}

Diante do que foi exposto, os autores em sua maioria constataram a partir de evidências científicas, que as plantas medicinais selecionadas neste trabalho apresentaram propriedade hipotensivas, embora reforcem a necessidade de realizar estudos mais aprofundados, visto que as plantas medicinais apresentam um complexo de compostos bioativos, sendo necessário isolar essas substâncias para estudos mais específicos e realizar além de ensaios pré-clínicos, os clínicos, o que carece de maiores investimentos. Além disso, foi possível constatar que as plantas medicinais apresentaram evidências científicas que podem servir de complemento terapêutico junto com os medicamentos convencionais no tratamento da hipertensão, ou até mesmo substituí-los em alguns casos, sob orientação médica. Porém, é necessário cautela, visto que apresentam efeitos adversos e interações medicamentosas, mesmo que em menor intensidade que os alopáticos, oferecendo riscos, principalmente aos idosos. Diante dessa relação de riscos e benefícios das plantas medicinais com ação antihipertensivas utilizada por idosos, foi possível evidenciar a importância da atenção farmacêutica, que através de métodos clínicos, em contado direto com o paciente, orienta sobre o uso racional das plantas medicinais e medidas não farmacológica no combate a hipertensão. Portanto, para que os farmacêuticos desempenhem essa função com excelência, é essencial que esse profissional se mantenha atualizado em relação a seus conhecimentos técnicos e científico nesta área de atuação. Espera-se que o presente trabalho colabore nesse aprimoramento profissional, destacando a importância da atenção farmacêutica na promoção, prevenção e recuperação da saúde de idosos com doenças crônicas, como a hipertensão. Nesse contexto, sugerimos mais pesquisas com evidencias científicas, que justifiquem o uso das espécies de plantas medicinais na prevenção e tratamento de doenças crônicas, destacando o papel da atenção farmacêutica que reúnam informações úteis aos profissionais de saúde para orientar o estudo, sensibilizar, levantar discussões e contribuir para promoção da utilização segura e racional das plantas medicinais. 


\section{Referências}

Abubakar, M. G., Ukwuani, A. N., \& Mande, U. U. (2015). Antihypertensive activity of Hibiscus Sabdariffa aqueous calyx extract in Albino rats. SKY journal of biochemistry, 4, 13-15.

Ajay, M., Chai, H. J., Mustafa, A. M., Gilani, A. H., \& Mustafa, M. R. (2007). Mechanisms of the anti-hypertensive effect of Hibiscus sabdariffa L. calyces. Journal of ethnopharmacology, 109(3), 388-393.

Al Disi, S. S., Anwar, M. A., \& Eid, A. H. (2016). Anti-hypertensive Herbs and their Mechanisms of Action: Part I. Frontiers in pharmacology, 6, 323.

Akinyemi, A., Thomé, G., Morsch, V., Stefanello, N., Goularte, J., Belló-Klein, A., Oboh, G., Rosa, M., Schetinger, C. (2015). Effect of dietary supplementation of ginger and turmeric rhizomes on angiotensin-1 converting enzyme (ACE) and arginase activities in L-NAME induced hypertensive rats. Journal of Functional Foods, 17, 792-801.

Anwar, M. A., Al Disi, S. S., \& Eid, A. H. (2016). Anti-Hypertensive Herbs and Their Mechanisms of Action: Part II. Frontiers in pharmacology, 7, 50.

Basati, G., Mehrabi Rad, F., Momeni Safarabadi, A., Shakib, P., Sedighi, M., \& Cheraghi, M. (2021). An Overview of the Most Important Medicinal Plants that Affect Hypertension and their Antihypertensive Mechanism. Journal of Medicinal plants and By-product, 10(Special), 1-8.

Brasil (2012). Ministério da saúde. Cadernos de atenção básica. Plantas medicinais e fitoterápico na atenção básica. http://bvsms.saude.gov.br/bvs/publicacoes/praticas_integrativas_complementares_plantas_medicinais_cab31.pdf

Chua, Y. T., Ang, X. L., Zhong, X. M., \& Khoo, K. S. (2015). Interaction between warfarin and Chinese herbal medicines. Singapore medical journal, 56(1), $11-18$.

Coon, J. T., \& Ernst, E. (2002). Panax ginseng: a systematic review of adverse effects and drug interactions. Drug safety, 25(5), 323-344.

Deka, A., \& Vita, J. A. (2011). Tea and cardiovascular disease. Pharmacological research, 64(2), 136-145.

Devi, R. C., Sim, S. M., \& Ismail, R. (2012). Effect of Cymbopogon citratus and Citral on Vascular Smooth Muscle of the Isolated Thoracic Rat Aorta. Evidence-based complementary and alternative medicine, 2012(1), 8.

Ekpenyong, C. E., Akpan, E., \& Nyoh, A. (2015). Ethnopharmacology, phytochemistry, and biological activities of Cymbopogon citratus (DC.) Stapf extracts. Chinese journal of natural medicines, 13(5), 321-337.

Fakeye, T. O., Pal, A., Bawankule, D. U., Yadav, N. P., \& Khanuja, S. P. (2009). Toxic effects of oral administration of extracts of dried calyx of Hibiscus sabdariffa Linn. (Malvaceae). Phytotherapy research: PTR, 23(3), 412-416.

Figueredo, C. A., Gurgel, I. G. D., \& Gurgel, G. D. (2014). A Política Nacional de Plantas Medicinais e Fitoterápicos: construção, perspectivas e desafios. Physis: Revista de Saúde Coletiva [online] 24(2). https://www.scielo.br/j/physis/a/fzMtXMF6QwLVHLk8nzxdFdM/abstract/?lang=pt

Gurley, B. J., Fifer, E. K., \& Gardner, Z. (2012). Pharmacokinetic herb-drug interactions (part 2): drug interactions involving popular botanical dietary supplements and their clinical relevance. Planta médica, 78(13), 1490-1514.

Herrera, A. A., Miranda, S. J., Avila, C. P., Herrera, A. S., Jiménez, F. J. E., Zamilpa, A., Román, R. R., Ponce, M. H., \& Tortoriello, J. (2007). Clinical effects produced by a standardized herbal medicinal product of Hibiscus sabdariffa on patients with hypertension. A randomized, double-blind, lisinopril-controlled clinical trial. Planta médica, 73(1), 6-12.

Hopkins, A. L., Lamm, M. G., Funk, J. L., \& Ritenbaugh, C. (2013). Hibiscus sabdariffa L. in the treatment of hypertension and hyperlipidemia: a comprehensive review of animal and human studies. Fitoterapia, 85, 84-94.

Hu, J., Webster, D., Cao, J., \& Shao, A. (2018). The safety of green tea and green tea extract consumption in adults - Results of a systematic review. Regulatory toxicology and pharmacology, 95, 412-433.

Isah T. (2015). Rethinking Ginkgo biloba L.: Medicinal uses and conservation. Pharmacognosy reviews, 9(18), 140-148.

Izzo, A. A., \& Ernst, E. (2009). Interações entre medicamentos fitoterápicos e medicamentos prescritos: uma revisão sistemática atualizada. Drugs, 69(13), $1777-1798$

Jacobs, L. P., Shaikh, K. A., Thomas, B., \& Nyakudya, T. T. (2020). An Overview of the Potential Use of Ethno-Medicinal Plants Targeting the ReninAngiotensin System in the Treatment of Hypertension. Molecules, 25(9), 2114.

Jang, S. J., Lim, H. J., \& Lim, D. Y. (2011). Inhibitory Effects of Total Ginseng Saponin on Catecholamine Secretion from the Perfused Adrenal Medulla of SHRs. Journal of ginseng research, 35(2), 176-190.

Jovanovski, E., Bateman, E. A., Bhardwaj, J., Fairgrieve, C., Mucalo, I., Jenkins, A. L., \& Vuksan, V. (2014). Effect of Rg3-enriched Korean red ginseng (Panax ginseng) on arterial stiffness and blood pressure in healthy individuals: a randomized controlled trial. Journal of the American Society of Hypertension, 8(8), 537-541.

Kim, J. H. (2012). Cardiovascular Diseases and Panax ginseng: A Review on Molecular Mechanisms and Medical Applications. Journal of ginseng research, $36(1), 16-26$.

Kim, J. H. (2018). Pharmacological and medical applications of Panax ginseng and ginsenosides: a review for use in cardiovascular diseases. Journal of ginseng research, 42(3), 264-269. 
Kolawole, J. A., \& Maduenyi, A. (2004). Effect of zobo drink (Hibiscus sabdariffa water extract) on the pharmacokinetics of acetaminophen in human volunteers. European journal of drug metabolism and pharmacokinetics, 29(1), 25-29.

Kouame, N.M., Kamagate, M., Koffi, C., Die-Kakou, H. M., Yao, N. A. R., Kakou, A. (2016). Cymbopogon citratus (DC.) Stapf : ethnopharmacologie, phytochimie, activités pharmacologiques et toxicologie. Phytothérapie, 14, 384-392.

Ku, D. D., Abdel, R. T. T., Dai, J., Kim-Park, S., Fallon, M. B., \& Abrams, G. A. (2002). Garlic and its active metabolite allicin produce endothelium- and nitric oxide-dependent relaxation in rat pulmonary arteries. Clinical and experimental pharmacology \& physiology, 29(1-2), 84-91.

Liu, L., Xu, F. R., \& Wang, Y. Z. (2020). Traditional uses, chemical diversity and biological activities of Panax L. (Araliaceae): A review. Journal of ethnopharmacology, 263, 112792 .

Li, W., Yang, J., Zhu, X. S., Li, S. C., \& Ho, P. C. (2016). Correlation between tea consumption and prevalence of hypertension among Singaporean Chinese residents aged $\geqslant 40$ years. Journal of human hypertension, 30(1), 11-17.

Locatelli, M., Zengin, G., Uysal, A., Carradori, S., De Luca, E., Bellagamba, G., Aktumsek, A., \& Lazarova, I. (2017). Multicomponent pattern and biological activities of seven Asphodeline taxa: potential sources of natural-functional ingredients for bioactive formulations. Journal of enzyme inhibition and medicinal chemistry, 32(1), 60-67.

Malati, C. Y., Robertson, S. M., Hunt, J. D., Chairez, C., Alfaro, R. M., Kovacs, J. A., \& Penzak, S. R. (2012). Influence of Panax ginseng on cytochrome P450 (CYP)3A and P-glycoprotein (P-gp) activity in healthy participants. Journal of clinical pharmacology, 52(6), 932-939.

Mansour, S. M., Bahgat, A. K., El-Khatib, A. S., \& Khayyal, M. T. (2011). Ginkgo biloba extract (EGb 761) normalizes hypertension in 2K, 1C hypertensive rats: role of antioxidant mechanisms, ACE inhibiting activity and improvement of endothelial dysfunction. Phytomedicine: international journal of phytotherapy and phytopharmacology, 18(8-9), 641-647.

Marques, L. A. M; Vale, F. V. V. R., Nogueira, V. A. S., Mialhe, F. L., \& Silva, L. C. (2011). Atenção farmacêutica e práticas integrativas e complementares no SUS: conhecimento e aceitação por parte da população sãojoanense. Physis: Revista de Saúde Coletiva, 21(2). https://www.scielo.br/j/physis/a/GQwvbNSDKrky5hpQ3t395hs/abstract/?lang=pt

Mashayekh, A., Pham, D. L., Yousem, D. M., Dizon, M., Barker, P. B., \& Lin, D. D. (2011). Effects of Ginkgo biloba on cerebral blood flow assessed by quantitative MR perfusion imaging: a pilot study. Neuroradiology, 53(3), 185-191.

Mesquita, T., de Jesus, I., Dos Santos, J. F., de Almeida, G., de Vasconcelos, C., Guatimosim, S., Macedo, F. N., Dos Santos, R. V., de Menezes-Filho, J., Miguel-Dos-Santos, R., Matos, P., Scalzo, S., Santana-Filho, V. J., Albuquerque-Júnior, R., Pereira-Filho, R. N., \& Lauton-Santos, S. (2017). Cardioprotective Action of Ginkgo biloba Extract against Sustained $\beta$-Adrenergic Stimulation Occurs via Activation of $\mathrm{M}_{2}$ /NO Pathway. Frontiers in pharmacology, 8, 220.

Mahmoudian-Sani, M. R., Hashemzadeh-Chaleshtori, M., Asadi-Samani, M., \& Yang, Q. (2017). Ginkgo biloba in the treatment of tinnitus: An updated literature review. The International Tinnitus Journal, 21(1), 58-62.

Mucalo, I., Jovanovski, E., Rahelić, D., Božikov, V., Romić, Z., \& Vuksan, V. (2013). Effect of American ginseng (Panax quinquefolius L.) on arterial stiffness in subjects with type-2 diabetes and concomitant hypertension. Journal of ethnopharmacology, 150(1), 148-153.

Nakasone, Y., Nakamura, Y., Yamamoto, T., \& Yamaguchi, H. (2013). Effect of a traditional Japanese garlic preparation on blood pressure in prehypertensive and mildly hypertensive adults. Experimental and therapeutic medicine, 5(2), 399-405.

Oliveira, I., Freitas, E., Detoni, K., \& Oliveira, D. (2017). Use of the patient's medication experience in pharmacists' decision making process. International Journal of Pharmacy, 7(1):1-8.

Oliveira, R. E. M., Filipin, M. D. V., \& Giardini, M. H. (2015). Intervenções farmacêuticas destinadas à otimização da adesão ao tratamento medicamentoso de um paciente. Revista Eletrônica De Farmácia, 12(2), 39-51.

Park, S. H., Huh, T. L., Kim, S. Y., Oh, M. R., Tirupathi Pichiah, P. B., Chae, S. W., \& Cha, Y. S. (2014). Antiobesity effect of Gynostemma pentaphyllum extract (actiponin): a randomized, double-blind, placebo-controlled trial. Obesity (Silver Spring, Md.), 22(1), 63-71.

Peng, X., Zhou, R., Wang, B., Yu, X., Yang, X., Liu, K., \& Mi, M. (2014). Effect of green tea consumption on blood pressure: a meta-analysis of 13 randomized controlled trials. Scientific reports, 4, 6251.

Pelentir, M., Deuschle, V. C. K. N; Deuschle, R. A. N. (2015). Importância da assistência e atenção farmacêutica no ambiente hospitalar. Ciência \& tecnologia, l(1), 20-28. http://www.revistaeletronicaocs.unicruz.edu.br/index.php/CIENCIAETECNOLOGIA/article/view/487

Pereira, A. S., Shitsuka, D. M., Parreira, F. J., \& Shitsuka, R. (2018). Metodologia da pesquisa científica. UFSM.

Ramalho, O. (2011). Atenção Farmacêutica: da Filosofia ao Gerenciamento da Terapia Medicamentosa. 1 ${ }^{\text {a. }}$. São Paulo: RNC Editora. 344 p.

Ried, K. (2016). Garlic Lowers Blood Pressure in Hypertensive Individuals, Regulates Serum Cholesterol, and Stimulates Immunity: An Updated Metaanalysis and Review. The Journal of nutrition, 146(2), 389S-396S.

Ryan, J. L., \& Morrow, G. R. (2010). Ginger. Oncology nurse edition, 24(2), 46-49.

Shaito, A., Thuan, D., Phu, H. T., Nguyen, T., Hasan, H., Halabi, S., Abdelhady, S., Nasrallah, G. K., Eid, A. H., \& Pintus, G. (2020). Herbal Medicine for Cardiovascular Diseases: Efficacy, Mechanisms, and Safety. Frontiers in pharmacology, 11, 422.

Shehabi, T., Iratni, R., \& Eid, A. H. (2016). Anti-atherosclerotic plants which modulate the phenotype of vascular smooth muscle cells. Phytomedicine, 23(11), $1068-1081$. 
Shin, W., Yoon, J., Oh, G.T., \& Ryoo, S. (2013). Korean red ginseng inhibits arginase and contributes to endotheliumdependent vasorelaxation through endothelial nitric oxide synthase coupling. Journal of Ginseng Research, 37(1):64.

Soares, A. J. S., Alkmim, A. C., Oliveira, D. R., Mendonça, S. A. M., \& Rodrigues, I. (2021). Potencialidades da prática da atenção farmacêutica no uso de fitoterápicos e plantas medicinais. Journal of Applied Pharmaceutical Sciences, (7), 10-21.

Stoner, G. D. (2013). Ginger: is it ready for prime time? Cancer prevention research (Philadelphia, Pa.), 6(4), 257-262.

Tajidin, E. (2012). Chemical composition and citral content in lemongrass (Cymbopogon citratus) essential oil at three maturity stages. African journal of biotechnology, 11(11), 2685-2693.

Tan, M.-S., Yu, J.-T., Tan, C.-C., Wang, H.-F., Meng, X.-F., Wang, C., Tan, L. (2015). Efficacy and adverse effects of ginkgo biloba for cognitive impairment and dementia: a systematic review and meta-analysis. Journal of Alzheimer's Disease, 43(2), 589-603.

Tavares, B., Silva, F., Luiza, M., Moura, C., Rego, G., Aquino, D. S. (2018). O papel do farmacêutico no controle da automedicação em idosos. Boletim Informativo Geum, 8(3), 18.

Telesi, E. (2016). Práticas integrativas e complementares em saúde, uma nova eficácia para o SUS. Estudos Avançados, 30(86), 99-112.

Ude, C., Schubert-Zsilavecz, M., \& Wurglics, M. (2013). Ginkgo biloba extracts: a review of the pharmacokinetics of the active ingredients. Clinical pharmacokinetics, 52(9), 727-749.

Varshney, R., \& Budoff, M. J. (2016). Garlic and Heart Disease. The Journal of nutrition, 146(2), 416S-421S.

Verma, T., Sinha, M., Bansal, N., Yadav, S. R., Shah, K., \& Chauhan, N. S. (2021). Plants Used as Antihypertensive. Natural products and bioprospecting, 11(2), 155-184.

Viana, S., Arantes, T., \& Ribeiro, S. (2017). Interventions of the clinical pharmacist in an Intermediate Care Unit for elderly patients. Einstein (São Paulo, Brazil), 15(3), 283-288.

WORLD HEALTH ORGANIZATION - WHO (2014). Global status report on noncommunicable diseases 2014 [Internet]. Geneva: World Health Organization. https://www.who.int/nmh/publications/ncd-status-report-2014/en/

Won, H. J., Kim, H. I., Park, T., Kim, H., Jo, K., Jeon, H., Ha, S. J., Hyun, J. M., Jeong, A., Kim, J. S., Park, Y. J., Eo, Y. H., \& Lee, J. (2019). Non-clinical pharmacokinetic behavior of ginsenosides. Journal of ginseng research, 43(3), 354-360.

Xiong, X. J., Liu, W., Yang, X. C., Feng, B., Zhang, Y. Q., Li, S. J., Li, X. K., \& Wang, J. (2014). Ginkgo biloba extract for essential hypertension: a systemic review. Phytomedicine: international journal of phytotherapy and phytopharmacology, 21(10), 1131-1136.

Yeh H., Chuang C., Chen H., Wan C., Chen T., Lin L. (2014). Bioactive components analysis of two various gingers (Zingiber officinale Roscoe) and antioxidant effect of ginger extracts. LWT - Food Science and Technology, 55(1), 329-334.

Yin, J. Y., Duan, S. Y., Liu, F. C., Yao, Q. K., Tu, S., Xu, Y., \& Pan, C. W. (2017). Blood Pressure Is Associated with Tea Consumption: A Cross-sectional Study in a Rural, Elderly Population of Jiangsu China. The journal of nutrition, health \& aging, 21(10), 1151-1159.

Wang, Y., Yu, H., Zhang, X., Feng, Q., Guo, X., Li, S., Li, R., Chu, D., \& Ma, Y. (2017). Evaluation of daily ginger consumption for the prevention of chronic diseases in adults: A cross-sectional study. Nutrition, 36, 79-84. 